Pp. 3-13

DOI: $10.1089 /$ cap.2014.0084

\title{
Clinical Evaluation of Youth with Pediatric Acute-Onset Neuropsychiatric Syndrome (PANS): Recommendations from the 2013 PANS Consensus Conference
}

\author{
Kiki Chang, MD,,* Jennifer Frankovich, MD, ${ }^{2, *}$ Michael Cooperstock, MD, MPH, ${ }^{3}$ \\ Madeleine W. Cunningham, $\mathrm{PhD}^{4}{ }^{4} \mathrm{M}$. Elizabeth Latimer, MD, ${ }^{5}$ Tanya K. Murphy, MD, \\ Mark Pasternack, MD, ${ }^{7}$ Margo Thienemann, MD, ${ }^{8}$ Kyle Williams, MD, ${ }^{9}$ Jolan Walter, MD, ${ }^{10}$ \\ and Susan E. Swedo, MD ${ }^{11}$; From the PANS Collaborative Consortium
}

\begin{abstract}
On May 23 and 24, 2013, the First PANS Consensus Conference was convened at Stanford University, calling together a geographically diverse group of clinicians and researchers from complementary fields of pediatrics: General and developmental pediatrics, infectious diseases, immunology, rheumatology, neurology, and child psychiatry. Participants were academicians with clinical and research interests in pediatric autoimmune neuropsychiatric disorder associated with streptococcus (PANDAS) in youth, and the larger category of pediatric acute-onset neuropsychiatric syndrome (PANS). The goals were to clarify the diagnostic boundaries of PANS, to develop systematic strategies for evaluation of suspected PANS cases, and to set forth the most urgently needed studies in this field. Presented here is a consensus statement proposing recommendations for the diagnostic evaluation of youth presenting with PANS.
\end{abstract}

\section{Background}

TN THE 1980s, investigators at the National Institutes of Health (NIH) noted a subset of children with obsessive-compulsive disorder (OCD) who had a sudden onset of their psychiatric symptoms, typically following infection with a variety of agents, including Streptococcus pyogenes, varicella, and Mycoplasma pneumoniae. These were termed pediatric infection triggered autoimmune neuropsychiatric disorders (PITANDS) (Allen et al. 1995). The investigators chose to focus on the subset of cases triggered by infections with group A Streptococcus (GAS) because of parallels between acute-onset OCD and the prodromal period of Sydenham chorea (SC), suggesting that acute-onset OCD might be a forme fruste of SC (Swedo et al. 1989; Swedo 1994; Swedo et al. 1994).

Systematic clinical investigations of SC and OCD led to discovery of a subgroup of OCD patients whose symptoms were trig- gered by GAS infections and labeled "pediatric autoimmune neuropsychiatric disorders associated with streptococcal infections" (PANDAS) (Swedo et al. 1998). The PANDAS subgroup is defined by an acute prepubertal onset of tics or OCD symptoms, association with GAS infection, and specific neuropsychiatric symptoms (Swedo et al. 1998, 2004; Murphy et al. 2012).

The requirement that GAS infections be associated with symptom onset/exacerbations proved difficult to operationalize, because of the prevalence of GAS infections in grade-school aged children, and the asymptomatic nature of rheumatogenic GAS organisms (Garvey et al. 1998); this resulted in both misdiagnoses and missed diagnoses of PANDAS (Gabbay et al. 2008). Additional problems were encountered in patients with tic disorders because the PANDAS subgroup is distinguished by an "abrupt onset and episodic course," but tics are frequently described as having an acute ("off/ on") onset and a waxing/waning course (Leckman et al. 2011). As a

\footnotetext{
${ }^{1}$ Professor of Psychiatry, Director of the Pediatric Bipolar Disorders Program, Stanford University School of Medicine, Stanford, CA.

${ }^{2}$ Clinical Assistant Professor of Pediatrics-Rheumatology, Stanford University School of Medicine, Stanford, California.

${ }^{3}$ Chief, Division of Infectious Diseases and Rheumatology, University of Missouri School of Medicine, Columbia, Missouri.

${ }^{4}$ Professor of Microbiology and Immunology, University of Oklahoma College of Medicine, Norman, Oklahoma.

${ }^{5}$ Pediatric Neurologist, Latimer Neurology Center, Bethesda, Maryland.

${ }^{6}$ Director and Professor of Pediatric Neuropsychiatry, Pediatrics and Psychiatry, University of South Florida, St Petersburg, Florida.

${ }^{7}$ Unit Chief of Pediatric Infectious Disease, Massachusetts General Hospital, Boston, Massachusetts.

${ }^{8}$ Associate Professor on the Adjunct Clinical Faculty, Stanford University School of Medicine, Stanford, California.

${ }^{9}$ Director of the Behavior and Immunology Clinic in the OCD and Related Disorders Program, Massachusetts General Hospital, Boston, Massachusetts.

${ }^{10}$ Director, Pediatric Immunodeficiency Program, Massachusetts General Hospital, Boston, Massachusetts.

${ }^{11}$ Chief, Pediatrics \& Developmental Neuroscience Branch, National Institute of Mental Health (NIMH), Rockville, Maryland.

*Co-first authors.
}

(C) The Author(s) 2015; Published by Mary Ann Liebert, Inc. This Open Access article is distributed under the terms of the Creative Commons Attribution Noncommercial License (http://creativecommons.org/licenses/by-nc/4.0/) which permits any noncommercial use, distribution, and reproduction in any medium, provided the original author(s) and the source are credited. 
result of the confusion surrounding the onset criteria, subsequent studies included youth likely to not meet criteria for PANDAS, and reported conflicting findings, making PANDAS an increasingly controversial diagnosis. Of greater concern, the criteria for PANDAS had been developed to define an etiologically homogeneous group of patients for research studies, and purposely excluded acute-onset cases not triggered by GAS infections, which inadvertently and unfortunately diverted attention from children with acute-onset OCD not related to GAS infections.

To address this, experts convened at the NIH in July 2010 and developed working criteria for pediatric acute-onset neuropsychiatric syndrome (PANS) (Swedo et al. 2012). Resulting PANS criteria describe a clinically distinct presentation, defined as follows.

I. Abrupt, dramatic onset of obsessive-compulsive disorder or severely restricted food intake

II. Concurrent presence of additional neuropsychiatric symptoms, (with similarly severe and acute onset), from at least two of the following seven categories:

1. Anxiety

2. Emotional lability and/or depression

3. Irritability, aggression, and/or severely oppositional behaviors

4. Behavioral (developmental) regression

5. Deterioration in school performance (related to attentiondeficit/hyperactivity disorder [ADHD]-like symptoms, memory deficits, cognitive changes)

6. Sensory or motor abnormalities

7. Somatic signs and symptoms, including sleep disturbances, enuresis, or urinary frequency

III. Symptoms are not better explained by a known neurologic or medical disorder, such as SC.

Many children with PANS are extremely ill, with extreme compulsions (licking shoes, barking), motor and phonic tics (whooping, wringing hands), behavioral regression, and terrifying episodes of extreme anxiety or aggression. The behavioral manifestations often prompt rapid referral to psychological or psychiatric services, but all patients should receive a full medical evaluation. It should be noted that PANS is a "diagnosis of exclusion" and that other known medical diseases must be ruled out before a diagnosis of PANS is assigned. By definition, the individual PANS symptoms overlap with a variety of psychiatric disorders, such as OCD, Tourette's syndrome, ADHD, depression, and bipolar disorder. However, the acuity of onset and simultaneous presentation of these symptoms differentiate PANS from these psychiatric conditions. The PANS diagnosis is, therefore, limited to cases with acute-onset symptoms in multiple domains. In some instances, children with PANS experience visual or auditory hallucinations; these cases deserve special note, as symptoms can appear identical to the psychotic symptoms seen in conditions such as schizophrenia, bipolar disorder, and lupus cerebritis. Again, because PANS is a diagnosis of exclusion, a comprehensive evaluation is needed to eliminate disorders presenting with similar neuropsychiatric symptoms. Here we provide consensus recommendations for the clinical and laboratory evaluation of youth with potential PANS. Treatment recommendations will be addressed in a future report.

\section{Diagnostic Evaluation}

When PANS is suspected, it is important to obtain a comprehensive medical and psychiatric history and perform a thorough
Table 1. Overview of PANS Evaluation

- Family history

- Medical history and physical examination

- Psychiatric evaluation

- Infectious disease evaluation

- Assessment of symptoms and history that points to need for further evaluation of immune dysregulation (autoimmune disease, inflammatory disease, immunodeficiency)

- Neurological assessment

- Assessment of somatic symptoms, including possible sleep evaluation

- Genetic evaluation

PANS, pediatric acute-onset neuropsychiatric syndrome.

physical examination. Table 1 provides an overview of the evaluation that should be conducted by the treating physician(s). If findings warrant, then relevant specialists should be consulted. Table 2 provides an overview of the differential diagnosis for PANS, reflecting the need to assess multiple medical and psychiatric domains.

\section{Family history}

The family history should include review of neurologic diseases, psychiatric disorders, autoimmune and autoinflammatory diseases, immunodeficiency syndromes, and frequent infections, including recurrent streptococcal pharyngitis (Table 3 ).

In one report, first-degree relatives of children with PANDAS were noted to have increased rates of OCD, tic disorders, and acute rheumatic fever, suggesting that children may have inherited a specific vulnerability to nonpyogenic poststreptococcal sequelae (Lougee et al. 2000). Maternal autoimmune diseases are also reported to be common among patients who meet criteria for PANDAS (Murphy 2010). It should be noted that neuropsychiatric disorders among siblings are particularly common and important to assess for as well. Psychiatric family history may provide important clues to genetic susceptibilities to $\mathrm{OCD}$, anxiety, or mood disorders, ADHD, pervasive developmental disorder (PDD), and others. It should be noted that a positive psychiatric family history does not preclude considering a diagnosis of PANS. In fact, a National Institute for Mental Health (NIMH) study found a 10 -fold increase in rates of OCD and tic disorders among first-degree relatives of PANDAS probands (Lougee et al. 2000).

\section{Medical history and physical examination}

The medical history and physical examination should address not only the signs and symptoms characteristic of PANS (such as

\section{Table 2. Differential Diagnosis for Youth with PANS}

- Obsessive compulsive disorder

- Anorexia nervosa

- Avoidant/restrictive food intake disorder (ARFID)

- Tourette syndrome

- Transient tic disorder

- Bipolar disorder

- Sydenham chorea

- Autoimmune encephalitis

- Systemic autoimmune disease ${ }^{\mathrm{a}}$

- Wilson's disease ${ }^{\mathrm{a}}$

${ }^{\mathrm{a}}$ Relatively rare conditions.

PANS, pediatric acute-onset neuropsychiatric syndrome. 
Table 3. Family History Assessment

FOR YOUTH WITH PANS (EXAMPles)

Neuro/Psychiatric

- Movement disorders: Tics, Tourette's syndrome, chorea

- Obsessive compulsive disorder, hoarding

- Anxiety, panic disorder, social phobia

- Eating disorder

- Alcoholism and other substance abuse or addiction problem

- Mood disorders: Depression, manic/depressive episodes/ bipolar disorder, rages, emotional lability

- Schizophrenia/psychotic disorders

- Autism/pervasive developmental disorder

- Attention-deficit/hyperactivity disorder (ADHD), learning disorders, intellectual disability

- Personality disorders

Autoimmune or autoinflammatory diseases

- Rheumatologic

- Rheumatic fever, heart valve replacement, Sydenham chorea

- Juvenile idiopathic arthritis, rheumatoid arthritis

Spondyloarthritis, reactive arthritis, enthesitis

- Connective tissue diseases including lupus, Sjögren's syndrome, scleroderma

- Kawasaki's disease, Henoch-Schonlein purpura, other vasculitis

- Familial Mediterranean fever, other recurrent fever syndromes

- Endocrinologic

Thyroiditis, Addison's disease, Type 1 diabetes

- Hematologic

- Idiopathic thrombocytopenia purpura (ITP), hemolytic anemia

- Antiphospholipid (APL) antibody syndrome

- Neurologic

- Guillain-Barré syndrome, transverse myelitis

- Multiple sclerosis or neurolmyelitis optica (Devic's disease)

- Acute disseminate encephalomyelitis (ADEM)

- Autoimmune encephalitis, brain vasculitis

- Gastroenterologic

- Celiac disease

- Inflammatory bowel disease (Crohn's disease, ulcerative colitis)

- Irritable bowel syndrome, food intolerances (i.e. gluten, dairy)

- Dermatologic

- Alopecia, vitiligo, psoriasis

Recurrent infections and immunodeficiency syndromes

- Recurrent infections: sinusitis, tonsilitis (especially strep), pneumonia, skin infections (i.e. staph), other infections.

- Chronic granulomatous diseases (CGD), common variable immunodeficiency (CVID), other immunodeficiency syndromes

Idiopathic diseases

- Chronic fatigue syndrome, fibromyalgia, and other pain disorders

PANS, pediatric acute-onset neuropsychiatric syndrome.

sensory abnormalities or choreiform movements), but also clinical features of disorders that must be excluded (SC, lupus, and Wilson's disease, among others). Therefore, the comprehensive evaluation must be individualized according to the patient's presentation and past medical history. Review of the child's medical records can help construct a timeline of early behavioral problems and childhood illnesses. The infection history of parents and siblings is of particular interest, regarding both susceptibility to, and potential sources of, PANS/PANDAS related infections (especially GAS infections).

PANS-related abnormalities that might be present on physical examination include: Dehydration or emaciation secondary to restricted intake of fluids or food; sequelae of compulsive behaviors (e.g., red ring around the mouth from excessive lip-licking, chapped hands from excessive washing or irritation of the external genitalia from excessive wiping); motor and/or phonic tics; evidence of sinusitis, chronic otitis, tonsillitis, or pharyngitis; and/or signs of GAS infection (i.e., pharyngitis, anal or vulvar redness, skin lesions).

Most youth with PANDAS have fairly normal neurological findings, but the frequency of neurological findings in youth with PANS is not well established. Choreiform movements, defined as fine piano-playing movements of the fingers when the child has arms and hands extended and eyes closed (Swedo et al. 1998, Touwen 1979) can be seen in children with PANS, but if full chorea is noted, workup for SC, antiphospholipid syndrome (APLS), lupus, and basal ganglia encephalitis should be pursued.

In addition to documenting physical evidence of PANS symptoms, a comprehensive review of systems and physical examination can help to exclude other medical conditions. The following is a list of the most pertinent PANS findings, signs of relevant rheumatogenic strep infection (known to trigger PANDAS and SC), and findings that are suggestive of other related conditions.

I. Constitutional symptoms (fevers, hair loss, weight loss, night sweats) that may indicate systemic autoimmune/ inflammatory disease, immunodeficiency, chronic infection, or thyroid disorder

II. Skin: Scarlatiniform rash (scarlet fever), erythema marginatum (acute rheumatic fever), malar rash (lupus), petechiae (APLS), chronic urticarial rash (vasculitis, other), livido reticularis (polyarteritis nodosa and other rheumatologic disorders), perianal redness (perianal strep).

III. Eyes: Dilated pupils or slow pupillary response to light examination (PANS and other neurological diseases), dark discoloration under eyes (allergies or chronic sinusitis), scleral injection (uveitis, episcleritis, or scleritis), or Kayser-Fleisher rings (Wilson's disease)

IV. Ear, nose, and throat: Recurrent, recent, or current tonsillitis, rhinosinusitis (chronic nasal congestion, nasal or postnasal discharge, sinus pressure or tenderness), hoarseness, otitis media, swollen nasal turbinates or "allergic salute" sign, petechiae on palate (APLS or Group A beta-hemolytic streptococci [GABHS] infection), or ulcer on palate (lupus)

V. Neck: Tymphadenopathy, thyromegaly, limited range of motion

VI. Chest: Chest pain, cough, dyspnea signs, rales (infection, rheumatologic disease), tachycardia, murmur or click, prolonged PR on electrocardiogram (ECG) (acute rheumatic fever, infection, other inflammatory disorder)

VII. Abdomen: Constipation, diarrhea, abdominal pain, abdominal tenderness, blood or mucous in stool, which may suggest underlying bowel disease

VIII. Musculoskeletal: Pain, warmth, tenderness, redness, pseudoparalysis (arthritis and acute rheumatic fever), myofascial tenderness or tender points (fibromyalgia)

IX. Neurological:

A. Cognition: PANS patients are frequently inattentive. If more severe impairment in cognition or memory is 
present, one should consider evaluation for inflammatory brain disease/autoimmune encephalitis.

B. Cranial nerves: These are typically normal in PANS patients.

C. Strength: PANS patients typically have normal strength but may exhibit mildly reduced proximal muscle weakness and slouched posture.

D. Fine motor skills: These can be normal or abnormal depending upon presence or absence of adventitious movements and developmental regression. Abnormalities may be elicited by having the child write, draw, or copy simple figures.

E. Abnormal movements: Motor or phonic tics are common in patients with PANS. Choreiform movements (piano playing fingers) may be present. These movements can be elicited with a Romberg test in which the child stands with the hands outstretched and eyes closed for 60 seconds. Hand movements are considered abnormal in children $>8$ years.

F. Reflexes: These can be normal or minimally depressed in the acute phase of PANS/PANDAS.

G. Cerebellar examination: This is normal in patients with PANS.

H. Gait: This is normal in patients with PANS unless compulsions or tics interrupt gait. Chorea may be elicited by stressed-gait evaluations and should prompt evaluation for SC.

Other positive neurological signs should be followed up with the appropriate tests or referral to a neurologist.

\section{Psychiatric evaluation}

A comprehensive psychiatric evaluation is important in order to understand the full range of psychiatric symptomatology, psychiatric history, developmental history, response to previous or current psychotropic medications, and response to past or current psychotherapy. Ideally, a child with PANS should be evaluated by an experienced child psychiatrist or psychologist. At minimum, however, primary care evaluation should include the full range of psychiatric and behavioral symptoms associated with PANS, including not only OCD and eating disorders, but also emotional lability, mood disorders, ADHD, anxiety disorders, tic disorders, psychosis, and neurodevelopmental disorders including autism spectrum disorder.

A structured diagnostic interview such as the Kiddie Schedule for Affective Disorders and Schizophrenia-Present and Lifetime (KSADS-PL) (Kaufman et al. 1997) may be useful, although these instruments were designed for research purposes and may be too cumbersome for clinical practice. If a structured interview is not used, it is particularly important to explore areas of potential embarrassment or sensitivity, including sexual or violent images and impulses, as these are common among patients with PANS (Frankovich et al. 2015a, in press). Self-injurious thoughts and behaviors also occur frequently and can be particularly worrisome in children with developmental regression and increased impulsivity. For example, children with PANS have made attempts at jumping out of a moving car or a second story window (Murphy et al. 2014).

\section{Mental status examination}

During an acute PANS episode, the child may appear hyperalert, unsmiling, anxious, and in the "fight or flight" mode. Parents may report that their child has a "terror-stricken look," although this is often present only in the first few days of illness. Memory impairments appear to be part of the PANS syndrome. Children often cannot recall details of their symptoms or their impact on functioning. Emotional lability (emotional incontinence) is a hallmark symptom of PANS and is characterized by involuntary and often uncontrollable episodes of crying or laughing that are often mood incongruent (i.e. a patient might laugh uncontrollably when angry or frustrated). Depression is also common, particularly during the later stages of the illness, so that the child may present with a flat or depressed affect. Agitation, irritability, aggression, and temper tantrums/rage episodes are also common. Speech is often affected, with a variety of notable observations, including "baby talk" secondary to developmental regression, a paucity of speech, (selective) mutism, or new onset of stuttering. Severe impulsivity and compulsive behavior may be present. Insight may be limited.

However, children with PANS will often willingly acknowledge the OCD thoughts once they are reassured that they will not have to reveal their content. Children may experience auditory or visual hallucinations, as well as violent imagery, and suicidal or homicidal ideation. These experiences should be assessed specifically. Children with PANS are often highly aware of their unwanted thoughts and actions, and may be embarrassed by them and apologetic afterward.

\section{General laboratory studies}

All patients meeting PANS criteria should have the following:

- Complete blood cell count with manual differential

- Erythrocyte sedimentation rate (ESR) and C-reactive protein (CRP)

- Comprehensive metabolic panel

- Urinalysis (to assess hydration) and to rule out inflammation for children with urinary complaints; clean-catch urine culture for those with pyuria

- Throat culture, anti-streptolysin O (ASO) and anti-DNAse B

The following laboratory tests should also be considered.

- If there are elevated inflammatory markers, fatigue, rashes, or joint pain, antinuclear antibody (ANA) or fluorescent antinuclear antibody (FANA) should be obtained; if ANA is elevated proceed with lupus workup.

- Antiphospholipid antibody work up should only be pursued if the patient has chorea, petechiae, migraines, stroke, thrombosis, thrombocytopenia, or levido rash. Workup includes: anticardiolipin antibody, dilute Russell's viper venom time (dRVVT), $\beta$ 2-glycoprotein I antibodies.

- If abnormal liver function tests or Kayser-Fleisher rings are present, there is a need to evaluate for Wilson's disease with ceruloplasmin and 24 urine copper tests.

\section{Infectious disease evaluation}

PANDAS. The diagnosis of PANDAS is based on evidence of recent or current streptococcal infection with onset or acute exacerbations. Streptococcal pharyngitis is confirmed with a properly performed throat culture or rapid antigen test. Rapid antigen tests are insufficiently sensitive; therefore, follow-up culture is required if the test is negative. Culture-proven GAS infection may also be documented at other symptomatic (to distinguish from asymptomatic sites in General laboratory studies section) sites, including the nasal cavity, skin and skin structure, and perianal or vaginal 
areas. Perianal streptococcal infection may accompany onset or exacerbation of neuropsychiatric symptoms (Toufexis et al. 2014). A history of scarlatiniform rash, impetigo, and perianal or vulvar dermatitis, or deep tissue GAS infection during the preceding 6 months should also be obtained. Culture of nasal secretions is advised, as a small fraction of sinusitis is caused by GAS (Cherry et al. 2014). Serologic diagnosis of recent GAS infection can be made by demonstrating a $0.2 \log _{10}$ rise (a $58 \%$ increase) in either ASO or ADB, ordinarily obtained 4-8 weeks apart, although only $62 \%$ of new GAS acquisitions were followed by such a rise (Johnson et al. 2010). A single high titer, rather than serial acute and convalescent titers, is not diagnostically reliable, but may be considered contributory if levels exceed twofold $\left(0.3 \log _{10}\right)$ above the laboratory's stated upper limit of normal, because these higher levels are uncommon in children without recent streptococcal infection.

Asymptomatic acquisition of GAS can initiate a rise in ASO or ADB (Johnson et al. 2010). It is not established whether such acquisition can initiate PANDAS in the absence of antimicrobial treatment, although analogous experience with rheumatic fever (Garvey et al. 1998), a prospective school-based study (Murphy et al. 2007) and our anecdotal experience support this potential.

Children with PANDAS, their family members, and other close contacts should be vigilantly observed for symptoms of pharyngitis and other streptococcal infections, with prompt clinical assessment and diagnostic testing when appropriate.

PANS. Most instances of PANS are suspected to be postinfectious in origin, although no single microbe other than GAS has yet been consistently associated with the onset of PANS. Therefore, a detailed review and documentation of associated febrile and nonfebrile infectious illnesses, including signs and symptoms and diagnostic testing, is advised. The most commonly observed antecedent infection seems to be upper respiratory infection, including rhinosinusitis, pharyngitis, or bronchitis. It is not yet clear if any one of those three presentations is more likely than the others to be associated with the initiation of PANS.

Mycoplasma pneumoniae has been associated with a number of postinfectious neurologic disorders (Muller et al. 2004; Walter et al. 2008), including a serologically diagnosed case closely resembling PANS (Ercan et al. 2008). It has also been documented anecdotally in a few cases by our group, and is therefore strongly suspected of being a stimulus for PANS. Because this organism is both persistent and treatable, the diagnosis may be pursued when a child with PANS or a family member has a history of cough, particularly cough lasting 1 week or more, with up to $\geq 4$ weeks between cases (American Academy of Pediatrics 2012). M. pneumoniae causes pharyngitis and tracheobronchitis more often than pneumonia, although it is unusual in rhinosinusitis. Both serologic testing and polymerase chain reaction (PCR) are commercially available. Like streptococcal serology, serologic diagnosis of $M$. pneumoniae is most accurate when rising titers are demonstrated in serial sera. Antibodies peak in 3-6 weeks. Single titer immunoglobulin (Ig)M serology, usually available as an enzyme immunoassay, is convenient, but subject to both false positives and false negatives. PCR is highly sensitive, and can be performed on sputum or a throat swab. It is most sensitive during the first 3 weeks of illness, but may also detect a carrier state for prolonged periods. A combination of both PCR for early diagnosis, and serology for later diagnosis, may, therefore, have the highest diagnostic yield (Waites et al. 2008). Both tests may be positive in children without apparent symptoms. It is not known whether asymptomatic colonization alone is sufficient to initiate PANS.
Influenza is associated with a number of PANS cases associated with well-documented acute influenza, including influenza H1N1. A characteristic syndrome in the patient or family member during a recognized epidemic period may be considered presumptive evidence of this disease; rapid testing during the acute illness is only $\sim 75 \%$ sensitive, whereas PCR testing is $>90 \%$ sensitive for detection of this disease.

Epstein Barr virus infection has been reported to precede various neuropsychological disorders (Caruso et al. 2000). We have observed several children with an acute neuropsychiatric disorder associated with serologically diagnosed Epstein Barr virus infection. These examples seem to be somewhat more complex than typical cases of PANS.

Borrelia burgdorferi (Lyme disease) is under suspicion because it has been associated with a wide range of postinfectious neuropsychiatric disorders, including a small number of cases with obsessivecompulsive disorder (Fallon et al. 1998). However no known cases of post-Lyme disease PANS have been noted. Diagnostic testing for Lyme disease, according to guidelines of the Centers for Disease Control (Centers for Disease Control and Prevention 2011) may be considered for children with PANS who: 1) Have a history of an illness compatible with prior clinical Lyme disease, and 2) live in regions in which the presence of Lyme disease is established. In the United States, this is almost exclusively limited to the six New England states, New York, New Jersey, Pennsylvania, Maryland, Virginia, Minnesota, and Wisconsin. Focal regions are also found in North Dakota, Iowa, Indiana, West Virginia, Illinois, and California (Centers for Disease Control and Prevention 2013).

As for other infectious disorders, we have anecdotally documented a few instances of herpes simplex infection and varicella related to PANS onset or flares. We believe that numerous other infectious agents, particularly those with characteristically prolonged colonization, have the potential to activate PANS as well. Appropriate documentation of such infections is warranted whenever suggested clinically.

\section{Evaluation for autoimmune and autoinflammatory diseases}

Neuropsychiatric symptoms can be caused by autoimmune encephalitis, systemic autoimmune disease, and other inflammatory diseases. Therefore, it is important to consider these etiologies. However, workup for these conditions should be done in a thoughtful manner, and only pursued if relevant symptoms are present. False positive antibody tests are common, and are meaningless if criteria for a clinical syndrome are not met. The identification of antibodies in patients can be very distressing for families, because the concept of false positivity can be difficult for a layperson to understand, especially where there is a severely ill child.

The diagnostic guidelines and indications for workup of autoimmune encephalitis are broad, and not within the scope of this article to discuss, but these conditions should be considered in cases in which one of the following symptoms is prominent: 1) Delirium, psychosis, and/or diffuse encephalopathy; 2) pervasive cognitive decline; 3 ) persistent memory impairment; 4) pervasive behavior deterioration; 5) seizures; and 6) movement abnormality not consistent with tics. The evaluation for autoimmune encephalitis includes neuroimaging, electroencephalogram (EEG), neuronal antibody testing (e.g., $N$-Methyl-D-aspartate [NMDA] receptor antibodies, voltage gated potassium channel antibodies) in serum and cerebrospinal fluid (CSF), thyroid antibodies 


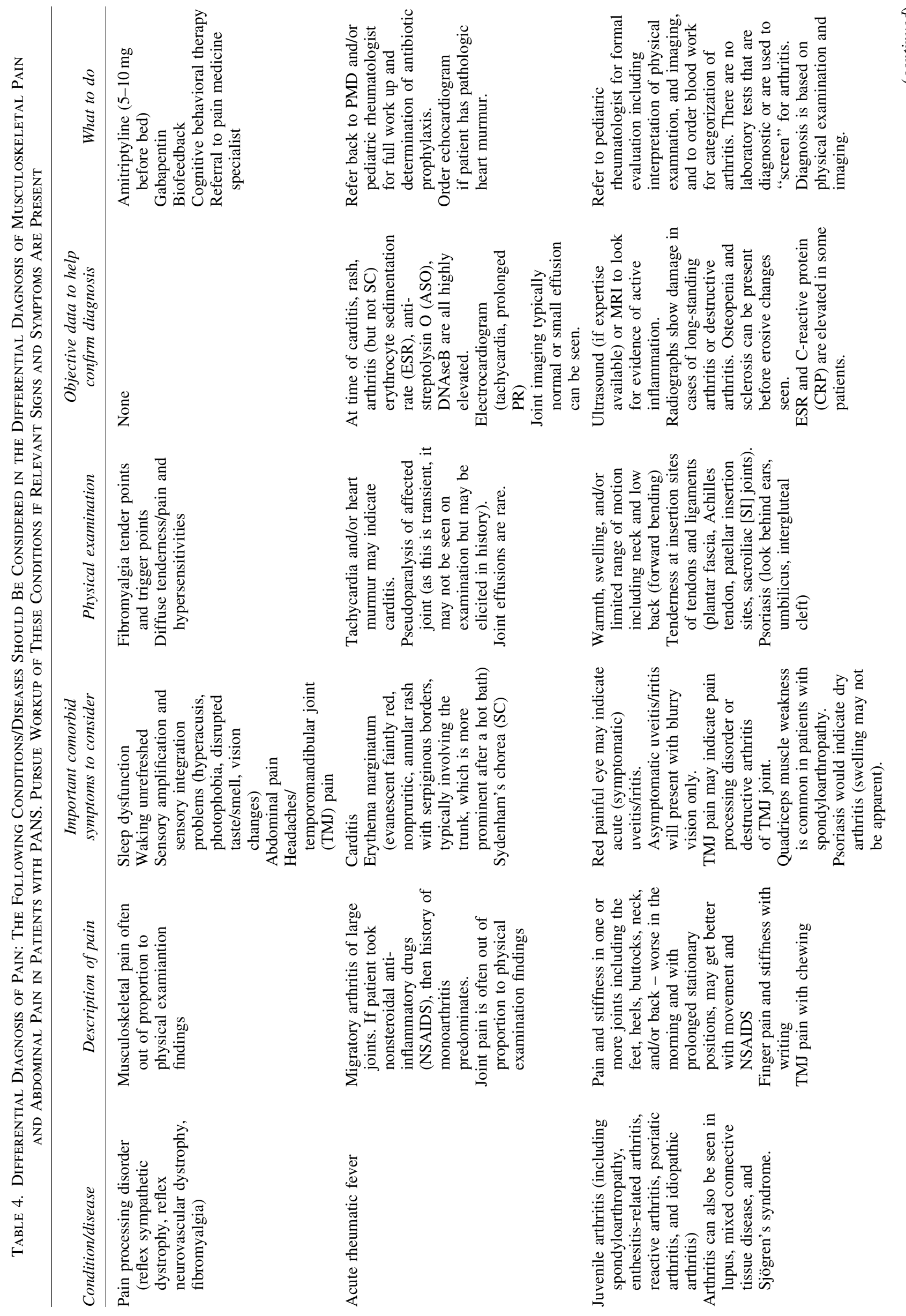




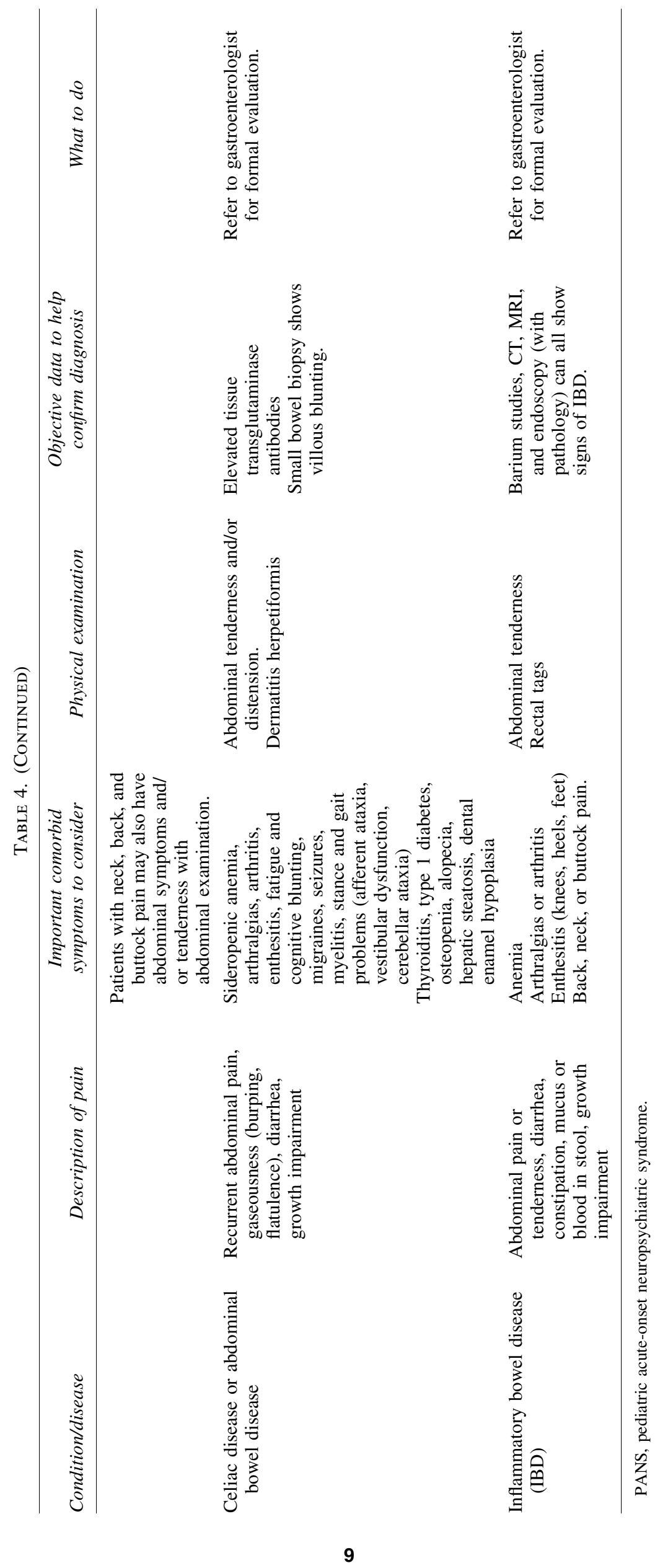


(thyroglobulin antibodies, thyroperoxidase antibodies), and paraneoplastic evaluations.

All patients should have a screening complete blood count with differential (CBC-D) (with peripheral blood smear), ESR, and CRP to evaluate for systemic inflammatory process. If significant anemia is present, further medical workup should include iron studies and consideration of inflammatory bowel disease or systemic autoimmune disease. Persistent thrombocytopenia and/or leukopenia may be a sign of systemic autoimmune diseases including lupus and other connective tissue diseases. Systemic lupus erythematosus (SLE), Sjögren's syndrome, and APLS can present with prominent neuropsychiatric symptoms including depression, headaches, seizures, psychosis, and movement disorders. OCD, eating restriction, and anxiety can be exacerbated by systemic illness (such as lupus) and severe anemia, and in patients experiencing a high level of stress from nonmedical causes.

If the patient presenting with PANS has elevated inflammatory markers (ESR or CRP) and/or cytopenias (low blood counts), and/ or dry eyes or dry mouth (not caused by medications) then a screening ANA test should be obtained. If the ANA is positive, then ANA subtiters (i.e., the ANA panel) should be ordered so that lupus-specific antibodies (double stranded [ds]DNA, Smith, RNP) and Sjögren's syndrome-related antibodies (SSA or anti-Ro and SSB or anti-La) may be detected. Additionally, if the ANA is positive, then obtaining complement levels (C3 and $\mathrm{C} 4)$ can also guide evaluation. If the patient shows evidence of a positive result on the ANA panel and low complement levels or systemic features, the patient should be referred to a pediatric rheumatologist. It is important to keep in mind that a positive ANA is found in 12-13\% of healthy children (Hilario et al. 2004; Satoh et al. 2012); therefore, a positive ANA alone does not warrant referral to rheumatology. The rate of positive ANAs in patients with PANDAS and PANS may be higher than the baseline in the pediatric population, an association that is currently undergoing additional examination.

Histone antibodies are associated with drug-induced lupus and lupus cerebritis, and have been reported to be positive in $17 \%$ of PANS patients (Frankovich et al. 2015b, in press). Although antihistone antibodies are of research interest, at this time they do not inform clinical management of PANS patients except in cases in which patients also meet criteria for SLE.

APL antibodies should be ordered in for patients with PANS if the patient has any of the following: Persistent thrombocytopenia, persistent petechiae, chorea, clinical thrombosis, migraines, strokes, or livedo reticularis. The APL workup is costly and should only be ordered if indicated. The APL workup includes the following: $\beta 2$ glycoprotein antibodies, anticardiolipin antibodies (IgG, IgM, IgA), DRVVT, and lupus anticoagulant. Abnormal results should be interpreted by a rheumatologist.

Behcet's disease can also present with neuropsychiatric symptoms, and should be considered in patients with recurrent oral and/or genital ulcers. It is necessary to examine the skin for signs of skin involvement (acneiform lesions, papulo-vesiculo-pustular eruptions, pseudofolliculitis, nodules, erythema nodosum, superficial thrombophlebitis, pyoderma gangrenosum-type lesions, erythema multiforme-like lesions, and palpable purpura), perform a pathergy test, consider HLA-B5 testing, and refer the patient to ophthalmology for evaluation of uveitis, as well as to rheumatology if criteria for Behcet's disease is met.

Patients meeting criteria for PANS commonly report pain, including chest pain, abdominal pain, headaches, musculoskeletal pain, and fatigue. Many of these patients meet criteria for a pain processing disorder (e.g., fibromyalgia) (Frankovich et al. 2015a, in press), and a minority have comorbid autoimmune/inflammatory disease. The psychiatric symptoms that PANS patients report are often so severe that they overshadow pain complaints. Therefore, we recommend asking about pain in all patients with PANS. Depending upon the pattern of pain and the physical examination, one whould consider the following causes of joint pain and/or abdominal pain: Acute rheumatic fever, juvenile arthritis, inflammatory bowel disease, and celiac disease. Table 4 provides details regarding the workup of pain in patients with PANS.

Immune complex panels and ferritin levels are of interest from the research standpoint, but at this juncture, these tests do not inform clinical management of patients with PANS unless diseasespecific symptoms are also present.

\section{Neural autoimmunity}

Testing for the presence of cross-reactive antineuronal antibodies may help guide diagnosis toward or away from PANS. Serum samples from acutely ill children with SC or PANDAS show elevated titers of antibodies against lysoganglioside (Kirvan et al. 2006a), tubulin (Kirvan et al. 2007), dopamine D2 receptor (Brimberg et al. 2012; Ben-Pazi et al. 2013; Cox et al. 2013), and dopamine D1 receptor (Ben-Pazi et al. 2013). More importantly, in both SC and PANDAS, the antibodies produced activation of calcium calmodulin protein kinase II (CaMK II) in the SK-N-SH human neuronal cell line (Kirvan et al. 2003, 2006b). Activation of CaMK II results in neuronal excitation and increased dopamine transmission (Kirvan et al. 2006b), which may be at least partially responsible for PANS symptoms. Recent studies have shown that $\mathrm{IgG}$ in youth with SC and PANDAS reacts with and signals the dopamine D2 receptor expressed in transfected cell lines (Cox et al. 2013). Similar results have also been shown for the D1 receptor expressed in transfected cell lines, but those data are not yet published (Cunningham, et al., unpublished data). Currently, Moleculera Labs (www.moleculera.com) is the only Clinical Laboratory Improvement Amendments (CLIA)-certified/Commission on Office Laboratory Accreditation (COLA)-accredited laboratory that provides testing for antitubulin, antilysoganglioside and antidopamine receptor antineuronal antibody titers by enzyme-linked immunosorbent assay (ELISA), as well as assays to measure CaMKII signaling. Although the Moleculera panel can provide useful ancillary information for children with suspected PANDAS, it is not yet clear that the assays are helpful for the larger cohort of children meeting PANS criteria (without GAS etiology). More research is needed to delineate the sensitivity and specificity of these tests for youth with PANS.

\section{Evaluation for immunodeficiency}

Some reports indicate that certain patients with Tourette syndrome, OCD and PANDAS have an increased tendency to develop infections or to show other evidence of immune dysfunction (Hornig 2013). Preliminary studies highlight altered immunoglobulin profiles (IgM, IgG subclasses, IgA) and decreased regulatory $\mathrm{T}$ cell count among this population compared with healthy controls (Kawikova et al. 2007, 2010; Bos-Veneman et al. 2011).

PANS patients should undergo an immunodeficiency assessment if there is a history of repeated infections, infection with an atypical organism or an unusual clinical course, first-degree family member with a history of overwhelming and/or fatal infection, or if the clinician is considering intravenous immunoglobulin 
(IVIG) treatment. Patients with solely autoimmune features should also have an immune evaluation, as autoimmunity may initially present as the sole feature of immune dysregulation.

Immunodeficiency screening should proceed in multiple steps, complemented with repeated clinical evaluation of the patient.

Initial workup should include the following:

I. Lymphocyte subsets (T, B, natural killer [NK] cells) with CBC with manual differential.

II. Quantitative immunoglobulins ( $\operatorname{IgG}, \operatorname{Ig}$ A, $\operatorname{IgM}, \operatorname{IgE}$ ) with IgG subclasses

III. Vaccine responses (Pneumococcus and tetanus antibody titers)

If the initial evaluation is reassuring, no further workup is needed. If the evaluation is abnormal and raises concerns about potential immune deficiency, the patient should be referred to an immunologist for further evaluation. Additional blood work may include: B and $\mathrm{T}$ cell maturation panel, postvaccination antibody titers at least 4 weeks following pneumococcal polysaccharide vaccine (Pneumovax 23 ), and $\mathrm{T}$ cell functional studies or any additional immune phenotyping relevant to the clinical history or prior laboratory findings.

\section{Additional diagnostic evaluations}

Brain MRIs are helpful when other conditions are suspected (e.g., central nervous system [CNS] small vessel vasculitis, limbic encephalitis) or when the patient has severe headaches, gait disturbances, cognitive deterioration, or psychosis. For particularly severe cases, an MRI with T-2 weighted images or contrast enhancement may be useful in demonstrating inflammatory changes in the basal ganglia, including volumetric changes (Giedd et al. 1996, 2000).

EEGs, particularly overnight evaluations, may be helpful in demonstrating focal or generalized slowing and/or epileptiform activity. These signs of abnormal brain activity or irritability were found in 7 of 42 (16\%) patients with PANDAS (Zhou et al. 2014). Similarly, polysomnography (PSG) also called "sleep studies" might reveal evidence of obstructive sleep apnea or abnormalities of sleep architecture, particularly in children with recent onset of insomnia or parasomnias (e.g., sleepwalking, night terrors). No data are yet available for results of PSG evaluations in PANS, but PSG studies in 11 children with PANDAS revealed parasomnias, periodic limb movements, and abnormalities of rapid eye movement (REM) sleep, including REM behavior disorder and nonspecific REM motor disinhibition (Hommer et al. 2014). If the PSG reveals specific abnormalities of sleep architecture, they may be targets of specific pharmacologic interventions, such as use of benzodiazepines for REM behavior disorder.

Lumbar puncture (LP) should be considered if there are MRI or EEG abnormalities, or encephalopathic symptoms such as delirium, alteration of consciousness, seizures, or psychosis. If an LP is done, the CSF evaluation should include oligoclonal bands, as well as standard measures such as glucose and protein. In addition, assays for antineuronal antibodies, such as anti-NMDA receptor antibodies, should be performed by a reliable laboratory (e.g., the Neuroimmunology Laboratory at Mayo Clinic Laboratories, directed by Dr. Vanda Lennon.)

Swallowing studies might be indicated for children with restricted food intake, particularly when related to fears of choking or vomiting. In SC, swallowing studies revealed dysphagia related to motor abnormalities and dyscoordination of the voluntary/involuntary muscles of the esophagus (Swedo et al. 1994).

\section{Discussion}

The recommended diagnostic workup for youth with suspected PANS presented here represents a consensus of the expert panel assembled. It is not intended to be algorithmic; therefore, clinicians should use these guidelines along with their best judgment. Although we tried to be as comprehensive as possible, we are aware that individual cases may require evaluation that is not mentioned here. As with all new presentations of psychiatric disease and/or escalations of psychiatric symptoms, investigation into the history must include the medical history (with emphasis on recent illnesses, exposures, fevers, history of painful joints, abdominal pain), review of systems (including discussion about pain and relevant rashes, such as scarlatina rash, erythema marginatum), and physical examnation (with focus on evaluating chorea and choreiform movements). Since this syndrome presents with prominent behavior regression and personality change, reactionary social disruptions (including issues with school functioning, friends, and parent accommodation) may give the appearance of there being a psychosocial cause to the illness. Acute-onset neuropsychiatric symptoms can be related to psychosocial traumas/stress, but trauma/stress response should be a diagnosis of exclusion. There is also clinical overlap between the symptoms of PANS and those of conversion disorder and psychosomatic illnesses. Therefore, a comprehensive history, psychiatric interview, and complete physical examination are critical in all cases of suspected PANS.

During the evaluation of children with PANS, it is particularly important to consider alternative medical explanations for the neuropsychiatric symptoms. Lupus cerebritis and the various autoimmune encephalitides may present with isolated cognitive and behavioral symptoms, personality change, and other symptoms (Dale and Brillot 2012). Referral to a neurologist or rheumatologist can be helpful in some cases, but should be focused on specific signs or symptoms of concern, as the subspecialists may not be experienced with the evaluation of psychiatric symptomatology. Therefore, the responsibility of evaluating PANS falls to primary care clinicians and child psychiatrists. In the future, empirically based algorithms for diagnosis and treatment will be developed. Until then, we hope that this article serves as a starting template for the proper evaluation of youth with PANS.

\section{Disclosures}

Dr. Chang is an unpaid consultant for GlaxoSmithKline, Lilly, and Bristol-Myers Squibb. He is on the DSMB for Sunovion. In the past two years he has received research support from GlaxoSmithKline and Merck. Dr. Walter is on the Advisory Board of Baxter regarding IGHy, a new formulation of subcutaneous immunoglobulin.

Dr. Madeleine Cunningham is co-founder and chief scientific officer of Moleculera Labs, which provides specialized antineuronal antibody testing. Several members of the PANS Collaborative Consortium have scientific collaborations with Dr. Cunningham. The other authors have nothing to disclose.

\section{References}

Allen AJ, Leonard HL, Swedo SE: Case study: A new infectiontriggered, autoimmune subtype of pediatric OCD and Tourette's syndrome. J Am Acad Child Adolesc Psychiatry 34:307-311, 1995. American Academy of Pediatrics: Mycoplasma pneumoniae and other mycoplasma species infections. In: Red Book 2012, Report of the Committee on Infectious Diseases, 29th ed., edited by L.K. Pick- 
ering. Elk Grove Village, IL: American Academy of Pediatrics, 518-521, 2012.

Ben-Pazi H, Stoner JA, Cunningham MW: Dopamine receptor autoantibodies correlate with symptoms in Sydenham's chorea. PLoS One 8:e73516, 2013.

Bos-Veneman NG, Olieman R, Tobiasova Z, Hoekstra PJ, Katsovich L, Bothwell AL, Leckman JF, Kawikova I: Altered immunoglobulin profiles in children with Tourette syndrome. Brain Behav Immun 25:532-538, 2011.

Brimberg L, Benhar I, Mascaro-Blanco A, Alvarez K, Lotan D, Winter C, Klein J, Moses AE, Somnier FE, Leckman JF, Swedo SE, Cunningham MW, Joel D: Behavioral, pharmacological, and immunological abnormalities after streptococcal exposure: a novel rat model of Sydenham chorea and related neuropsychiatric disorders. Neuropsychopharmacology 37:2076-2087, 2012.

Caruso JM, Tung GA, Gascon GG, Rogg J, Davis L, Brown WD: Persistent preceding focal neurologic deficits in children with chronic Epstein-Barr virus encephalitis. J Child Neurol 15:791-796, 2000.

Centers for Disease Control and Prevention: Lyme Disease, Two-Step Laboratory Testing Process, 2011. Available at http://www.cdc.gov/ lyme/diagnosistesting/LabTest/TwoStep/index.html. Accessed June, 2014.

Centers for Disease Control and Prevention: Lyme Disease (map), 2013. Available at http://www.cdc.gov/lyme/stats/maps/map2012 .html. Accessed August, 2014.

Cherry J, Mundi J and Shapiro N: Rhinosinusitis. In: Feigin and Cherry's Textbook of Pediatric Infectious Diseases, 7th ed., edited by J.D. Cherry, G.J. Harrison, S.L. Kaplan, W.J. Steinbach, and P.J. Hotez. Philadelphia: Elsevier Saunders, 193-203, 2014.

Cox CJ, Sharma M, Leckman JF, Zuccolo J, Zuccolo A, Kovoor A, Swedo SE, Cunningham MW: Brain human monoclonal autoantibody from sydenham chorea targets dopaminergic neurons in transgenic mice and signals dopamine $\mathrm{d} 2$ receptor: Implications in human disease. J Immunol 191:5524-5541, 2013.

Dale RC, Brilot F: Autoimmune basal ganglia disorders. J Child Neurol 27:1470-1481, 2012.

Ercan TE, Ercan G, Severge B, Arpaozu M, Karasu G: Mycoplasma pneumoniae infection and obsessive-compulsive disease: a case report. J Child Neurol 23:338-340, 2008.

Fallon BA, Kochevar JM, Gaito A, Nields JA: The underdiagnosis of neuropsychiatric Lyme disease in children and adults. Psychiatr Clin North Am 21:693-703, viii, 1998.

Frankovich J, Thienemann M, Rana S, Chang, K: Five youth with pediatric acute-onset neuropsychiatric syndrome of differing etiologies. J Child Adolesc Psychopharm 15:31-37, 2015 a.

Frankovich J, Thienemann M, Pearlstein J, Crable A, Brown K, Chang K: Multidisciplinary Clinic Dedicated to Treating Youth with Pediatric Acute-Onset Neuropsychiatric Syndrome: Presenting Characteristics of the First 47 Consecutive Patients. Child Adolesc Psycopharm 25:38-47, 2015b.

Gabbay V, Coffey BJ, Babb JS, Meyer L, Wachtel C, Anam S, Rabinovitz B: Pediatric autoimmune neuropsychiatric disorders associated with streptococcus: Comparison of diagnosis and treatment in the community and at a specialty clinic. Pediatrics $122: 273-278$, 2008.

Garvey MA, Giedd J, Swedo SE: PANDAS: The search for environmental triggers of pediatric neuropsychiatric disorders. Lessons from rheumatic fever. J Child Neurol 13:413-423, 1998.

Giedd JN, Rapoport JL, Garvey MA, Perlmutter S, Swedo SE: MRI assessment of children with obsessive-compulsive disorder or tics associated with streptococcal infection. Am J Psychiatry 157:281283, 2000.

Giedd JN, Rapoport JL, Leonard HL, Richter D, Swedo SE: Case study: Acute basal ganglia enlargement and obsessive-compulsive symptoms in an adolescent boy. J Am Acad Child Adolesc Psychiatry 35:913-915, 1996.

Hilario MO, Len CA, Roja SC, Terreri MT, Almeida G, Andrade LE: Frequency of antinuclear antibodies in healthy children and adolescents. Clin Pediatr (Phila) 43:637-642, 2004.

Hommer R, Buckley A, Swedo S: New onset sleep disturbance and PSG findings in children with acute or subacute neuropsychiatric changes. Abstract submitted to 2014 AACAP annual meeting, 2014.

Hornig M: The role of microbes and autoimmunity in the pathogenesis of neuropsychiatric illness. Curr Opin Rheumatol 25:488-795, 2013.

Johnson DR, Kurlan R, Leckman J, Kaplan EL: The human immune response to streptococcal extracellular antigens: Clinical, diagnostic, and potential pathogenetic implications. Clin Infect Dis 50:481-490, 2010.

Kaufman J, Birmaher B, Brent D, Rao U, Flynn C, Moreci P, Williamson D, Ryan N: Schedule for Affective Disorders and Schizophrenia for School-Age Children-Present and Lifetime Version (K-SADS-PL): Initial reliability and validity data. J Am Acad Child Adolesc Psychiatry 36:980-988, 1997.

Kawikova I, Grady BP, Tobiasova Z, Zhang Y, Vojdani A, Katsovich L, Richmand BJ, Park TW, Bothwell AL, Leckman JF: Children with Tourette's syndrome may suffer immunoglobulin A dysgammaglobulinemia: Preliminary report. Biol Psychiatry 67:679-683, 2010.

Kawikova I, Leckman JF, Kronig H, Katsovich L, Bessen DE, Ghebremichael M, Bothwell AL: Decreased numbers of regulatory T cells suggest impaired immune tolerance in children with Tourette syndrome: A preliminary study. Biol Psychiatry 61:273-278, 2007.

Kirvan CA, Cox CJ, Swedo SE, Cunningham MW: Tubulin is a neuronal target of autoantibodies in Sydenham's chorea. J Immunol 178:7412-7421, 2007.

Kirvan CA, Swedo SE, Heuser JS, Cunningham MW: Mimicry and autoantibody-mediated neuronal cell signaling in Sydenham chorea. Nat Med 9:914-920, 2003.

Kirvan CA, Swedo SE, Kurahara D, Cunningham MW: Streptococcal mimicry and antibody-mediated cell signaling in the pathogenesis of Sydenham's chorea. Autoimmunity 39:21-29, 2006a.

Kirvan CA, Swedo SE, Snider LA, Cunningham MW: Antibodymediated neuronal cell signaling in behavior and movement disorders. J Neuroimmunol 179:173-179, 2006b.

Leckman JF, King RA, Gilbert DL, Coffey BJ, Singer HS, Dure LSt, Grantz H, Katsovich L, Lin H, Lombroso PJ, Kawikova I, Johnson DR, Kurlan RM, Kaplan EL: Streptococcal upper respiratory tract infections and exacerbations of tic and obsessive-compulsive symptoms: A prospective longitudinal study. J Am Acad Child Adolesc Psychiatry 50:108-118 e103, 2011.

Lougee L, Perlmutter SJ, Nicolson R, Garvey MA, Swedo SE: Psychiatric disorders in first-degree relatives of children with pediatric autoimmune neuropsychiatric disorders associated with streptococcal infections (PANDAS). J Am Acad Child Adolesc Psychiatry 39:1120-1126, 2000.

Muller N, Riedel M, Blendinger C, Oberle K, Jacobs E, Abele-Horn M: Mycoplasma pneumoniae infection and Tourette's syndrome. Psychiatry Res 129:119-125, 2004.

Murphy TK, Snider LA, Mutch PJ, Harden E, Zaytoun A, Edge PJ, Storch EA, Yang MC, Mann G, Goodman WK, Swedo SE: Relationship of movements and behaviors to Group A Streptococcus infections in elementary school children. Biol Psychiatry 61:279-284, 2007.

Murphy TK, Storch EA, Lewin AB, Edge PJ, Goodman WK: Clinical factors associated with pediatric autoimmune neuropsychiatric disorders associated with streptococcal infections. J Pediatr 160:314-319, 2012.

Murphy TK, Patel PD, McGuire JF, Kennel A, Mutch PJ, Athill EP, Hanks CE, Lewin AB, Storch EA, Toufexis MD, Dadlani GH, Rodriquez CA: Characterization of the pediatric acute-onsete neuropsychiatric syndrome phenotype. J Child Adolesc Psychopharm 15:14-25, 2015. 
Satoh M, Chan EK, Ho LA, Rose KM, Parks CG, Cohn RD, Jusko TA, Walker NJ, Germolec DR, Whitt IZ, Crockett PW, Pauley BA, Chan JY, Ross SJ, Birnbaum LS, Zeldin DC, Miller FW: Prevalence and sociodemographic correlates of antinuclear antibodies in the United States. Arthritis Rheum 64:2319-2327, 2012.

Swedo S, Leckman J, Rose N: From research subgroup to clinical syndrome: Modifying the PANDAS criteria to describe PANS (pediatric acute-onset neuropsychiatric syndrome). Pediatr Therapeutics 2:1-8, 2012.

Swedo SE: Sydenham's chorea. A model for childhood autoimmune neuropsychiatric disorders. JAMA 272:1788-1791, 1994.

Swedo SE, Leonard HL, Garvey M, Mittleman B, Allen AJ, Perlmutter S, Dow S, Zamkoff J, Dubbert BK, Lougee L: Pediatric autoimmune neuropsychiatric disorders associated with streptococcal infections: Clinical description of the first 50 cases. Am J Psychiatry 155:264-271, 1998.

Swedo SE, Leonard HL, Kiessling LS: Speculations on antineuronal antibody-mediated neuropsychiatric disorders of childhood. Pediatrics 93:323-326, 1994.

Swedo SE, Leonard HL, Rapoport JL: The pediatric autoimmune neuropsychiatric disorders associated with streptococcal infection (PANDAS) subgroup: separating fact from fiction. Pediatrics 113:907-911, 2004.

Swedo SE, Rapoport JL, Leonard H, Lenane M, Cheslow D: Obsessive-compulsive disorder in children and adolescents. Clinical phenomenology of 70 consecutive cases. Arch Gen Psychiatry 46:335-341, 1989.

Toufexis M, Deoleo C, Elia J, Murphy TK: A link between perianal strep and pediatric autoimmune neuropsychiatric disorder associ- ated with streptococcal infection (PANDAS). J Neuropsychiatry Clin Neurosci 26:164-168, 2014.

Touwen B: Examination of the Child With Minor Neurological Dysfunction: Clinics in Developmental Medicine No 71. London: SIMP, Heinemann; 1979.

Waites KB, Balish MF, Atkinson TP: New insights into the pathogenesis and detection of Mycoplasma pneumoniae infections. Future Microbiol 3:635-648, 2008.

Walter ND, Grant GB, Bandy U, Alexander NE, Winchell JM, Jordan HT, Sejvar JJ, Hicks LA, Gifford DR, Alexander NT, Thurman KA, Schwartz SB, Dennehy PH, Khetsuriani N, Fields BS, Dillon MT, Erdman DD, Whitney CG, Moore MR: Community outbreak of Mycoplasma pneumoniae infection: School-based cluster of neurologic disease associated with household transmission of respiratory illness. J Infect Dis 198:1365-1374, 2008.

Zhou X, Buckley A, Swedo S, Inati S: Epileptiform abnormalities in pediatric autoimmune neuropsychiatric disorders associated with streptococcal infections (PANDAS) patients. 2014.

Address correspondence to:

Kiki D. Chang, $M D$ Stanford University School of Medicine Division of Child and Adolescent Psychiatry 401 Quarry Road Stanford, CA 94305-5540

E-mail: kchang88@stanford.edu 